\title{
Estimation of the plasma blob occurrence rate
}

\author{
R. Häcker ${ }^{1,2}$, G. Fuchert ${ }^{3}$, D. Carralero ${ }^{2,4}$, P. Manz ${ }^{2}$ \\ 1 Physik-Department E28, Technische Universität München, \\ James-Franck-Str. 1, 85748 Garching, Germany \\ 2 Max Planck Institute for Plasma Physics, Boltzmannstr.2 ,85748 Garching, Germany \\ 3 Max Planck Institute for Plasma Physics, Wendelsteinstr. 1, 17491 Greifswald, Germany \\ ${ }^{4}$ CIEMAT - Laboratorio Nacional de Fusión, Avda. Complutense 40, 28040 Madrid, Spain
}

(Dated: January 6, 2018)

\begin{abstract}
As part of the blob duty cycle, the blob detection rate, also called the blob occurrence rate or simply blob frequency, is of critical importance to predict the impact of plasma blobs on plasma wall interaction and modification of the scrape-off layer such as the scrape-off layer width. This rate is estimated based on extrapolation from measurements on present devices. A recent physics-based prediction of the blob detection rate (G. Fuchert et al. Plasma Physics Controlled Fusion 58, 054006 (2016)) has been compared by means of gyrofluid simulations with varying collisionality and shear layer strength. The prediction shows a robust agreement within a factor of two despite varying generation positions and driving instabilities.
\end{abstract}

PACS numbers:

\section{INTRODUCTION}

Transport in the scrape-off layer (SOL) of magnetically confined fusion experiments is determined to a huge fraction by filamentary structures of enhanced plasma pressure, also called blobs [1-3]. A lot of progress has been made in understanding the physics of blobs. In particular the relation between blob size $\delta_{b}$ and velocity $v_{b}$ has been studied extensively [4-11]. However, it has still been unknown or there is no general agreement on how, where and at which rate blobs are generated. The prediction of the blob detection rate $f_{d}[12]$, also called the blob occurrence rate [13] or simply blob frequency [14], as part of the blob duty cycle $f_{d} \tau_{b}$ or blob packing fraction is of great use for practical considerations about the blob transport, the SOL width [13, 15] and plasma-wall interaction $[14,15]$, since especially the large amplitude events carry the most energy and particles. Whereas the correlation time of the blob $\tau_{b} \sim \delta_{b} / v_{b}$ can be related to reasonably well understood quantities, the blob detection rate has to be estimated by experience from devices currently in operation [13-15], but not from a predictive formula. A recently developed model enables the quantitative prediction of the blob detection rate in a fusion plasma [12].

In the following, the basic idea of the model presented in Ref. [12] is summarized: There is a primal region of blob generation, where a dominant instability generates seed fluctuations. An instability with a wave length $\lambda_{\perp}$ in the binormal direction propagates with its binormal phase velocity $v_{p h}$ and is additionally advected by the binormal background $E \times B$ velocity $v_{E \times B}$. Therefore such an instability can excite seed fluctuations at a rate $[12]$

$$
f_{g}=\frac{\left(v_{E \times B}+v_{p h}\right)}{\lambda_{\perp}} .
$$

There exists a typical blob size $\delta_{b}$, where coherent blobs can form [16] which are most stable against secondary instabilities (Kelvin-Helmholtz and interchange instability) $[17,18]$. Therefore, the most stable blob size defines a selection rule on which scale the seed fluctuations can develop to blobs. The conversion between the poloidal blob size $\delta_{b}$, defined as the full width (in the binormal direction) at half maximum (FWHM) of the density perturbation, and the poloidal wave length $\lambda_{\perp}$ of the dominant edge instability $\lambda_{\perp}=3 \delta_{b}$ has been used, since the FWHM of the positive part of a sine wave is approximately $\lambda_{\perp} / 3[12]$. The most stable blob size can be inferred by model predictions $[4,6]$. The blob detection rate

$$
f_{d}=f_{g} \gamma_{d}
$$

is predicted to depend on the blob generation rate and the amplitude distribution function by

$$
\gamma_{\mathrm{d}}=1-\frac{\left.\mathrm{CDF}\right|_{0} ^{a_{\text {thresh }}}}{\left.\mathrm{CDF}\right|_{0} ^{\infty}}
$$

where $\left.\mathrm{CDF}\right|_{a} ^{b}$ (cumulative distribution function) denotes the integral of the distribution from $a$ to $b$. Whereas the amplitude distribution function in the SOL tend to follow a Gamma distribution [3, 19-21], the amplitude distribution function at the generation position does not necessarily have to be Gamma distributed. Turbulence is expected in general to exhibit a close to Gaussian statistics. In ASDEX Upgrade, JET and Alcator C-Mod close to the separatrix the distribution function is close to Gaussian [22-24]. The Gamma distribution is very similar to a Gaussian when the shape parameter is large. Assuming a Gaussian distribution and $a_{\text {thresh }}=2.5 \sigma$ (with the standard deviation $\sigma$ of the fluctuation signal), $\gamma_{\mathrm{d}}^{\text {gauss }} \approx 0.0124$ is obtained. In case of an exponential distribution, recently discussed in SOL plasmas in Ref. [25], $\gamma_{\mathrm{d}}^{\exp } \approx 0.0821$. The model depends on the profiles by the $E \times B$ velocity in Eq. (1), on the underlying instability by the phase velocity in Eq. (1), on the blob model 
due to $\lambda_{\perp}$ in Eq. (1) and on the distribution function in $\gamma_{d}$ in Eq. (2). Therefore, the model can be regarded as modular in the sense that it generalizes about the type of instability triggering blob generation and the relevant blob dynamics. This modularity is necessary, because there is no entire agreement on which instability may be responsible for blob generation, or which distribution function to use. A more detailed discussion can be found in Ref. [12].

In the present contribution the basic idea of Ref. [12] is studied with the help of the simulations. To account for the modularity of the model, the simulations cover different scenarios realized by a density scan exhibiting variations in collisionality, shear layer strength and dominant instability. Details on the simulation set are given in the following section. Although the simulated density scan qualitatively recover most of the features of a typical density-ramp up discharge, the quantitative agreement leaves room for improvement. A detailed study of the physics of the density ramp-up scenario with optimized simulation parameters is left for future work.

\section{GEMR SIMULATIONS}

Simulations have been carried out with the threedimensional gyrofluid electromagnetic turbulence model GEMR [26, 27]. Within this framework, experimental and modeling results have already been compared with reasonably good agreement [28-31]. A circular plasma cross-section with toroidal axisymmetry is assumed. The coordinate system is aligned with the equilibrium magnetic field. The set of grid points with constant parallel coordinate represents the drift plane, which is perpendicular to the magnetic field. The open field lines corresponding to the SOL are implemented by the boundary conditions via a perturbed Debye sheath current $[32,33]$. As GEMR is a $3 \mathrm{D}$ code no parallel closure is preassigned. Although being a $\delta$-f limited code the gradients evolve freely, as required by the strength of fluctuating dynamics in this plasma region. The profiles are included in the dependent variables also in the polarization, therefore GEMR is a global model. Details on the self-consistent treatment of the profiles and MHD equilibrium can be found in Ref. [26].

The GEMR simulations are adjusted to ASDEX Upgrade parameters (major radius $R=1.65 \mathrm{~m}$, minor radius $a=0.5 \mathrm{~m}$ and magnetic field strength $B=2.5 \mathrm{~T}$ ). The coordinate system $(x, y, s)$ is in the radial, binormal and parallel direction to the magnetic field. The simulations are performed on a $128 \times 512 \times 16$ grid, where only the drift plane $(128 \times 512)$ at the outboard midplane is analyzed here. The simulations cover the region $0.95<\rho<1.05$ corresponding to about $2.5 \mathrm{~cm}$ inside to $2.5 \mathrm{~cm}$ outside the LCFS. The radial and binormal resolution of the drift plane is roughly $0.3 \rho_{s}$ and $0.5 \rho_{s}$, respectively. About $1 \mathrm{~ms}$ is simulated in the saturated phase. To study the density dependence the initial

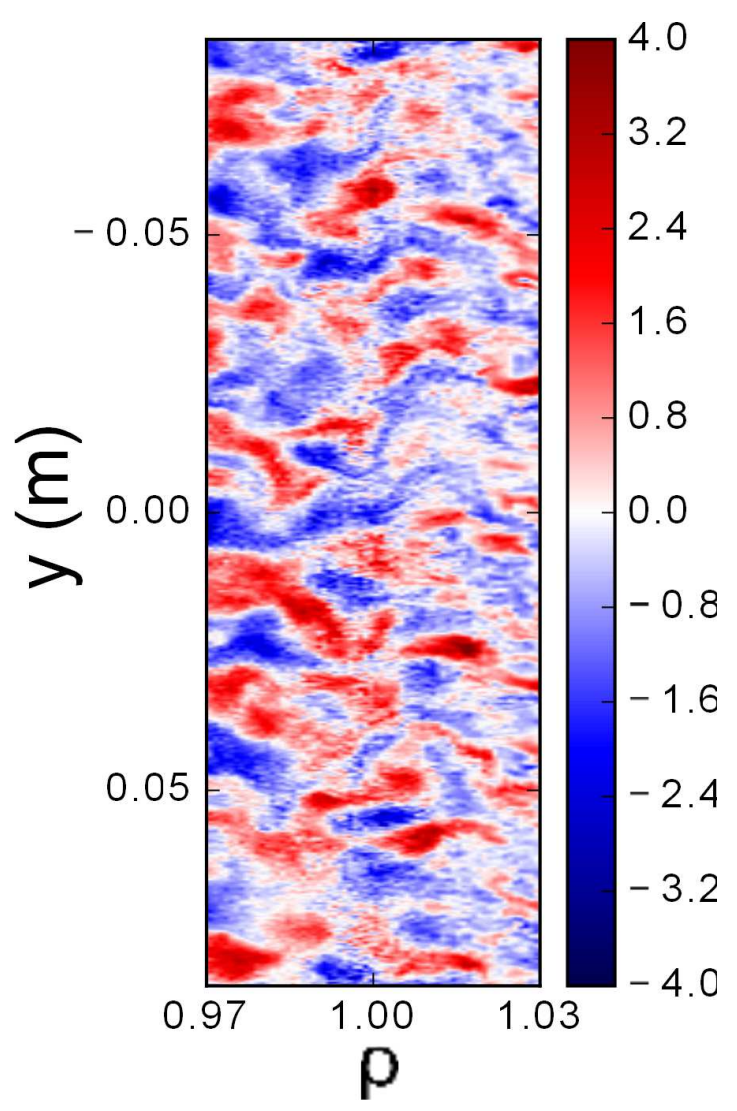

FIG. 1: Snapshot of the drift-plane at the outer midplane at low density $\left(n_{e}=2.6 \cdot 10^{18} \mathrm{~m}^{-3}\right)$. Red (blue) color indicates a positive (negative) density fluctuation. Values are given in units of the local standard deviations.

temperature is kept the same for all simulations (at the LCFS as the reference electron and ion temperature of $T_{e}=T_{i}=60 \mathrm{eV}$ and initial temperature gradient lengths of $L_{T e}=L_{T_{i}}=4.5 \mathrm{~cm}$ ). The initial density gradient length in the confined plasma is also kept the same for all simulations at $L_{n}=9 \mathrm{~cm}$. The effective charge is $Z_{\text {eff }}=2$ and the safety factor is $q=4.6$. Densities are given by at their LCFS value in this manuscript. The density at the LCFS varies between $3 \cdot 10^{18} \mathrm{~m}^{-3}$ and $4.5 \cdot 10^{19} \mathrm{~m}^{-3}$. The Greenwald density for these plasmas is $n_{G}=5.2 \cdot 10^{19} \mathrm{~m}^{-3}$, therefore the LCFS density varies from $5 \%$ to $85 \%$ of the Greenwald density.

To illustrate the dynamics, two snapshots of the driftplane at the outer midplane are shown. At low densities (Fig. 1) a process is revealed, where blobs in and outside the confined region merge and thereby transfer density into the SOL [34]. This exemplifies the picture of turbulence spreading, where turbulent structures radially transport energy by merging and breaking up. At high densities (Fig. 2) much larger blobs are observed, which are propagating mostly radial from inside to outside of the simulation domain. However, they still undergo multiple interactions on their path.

The control parameters for the density dependence 


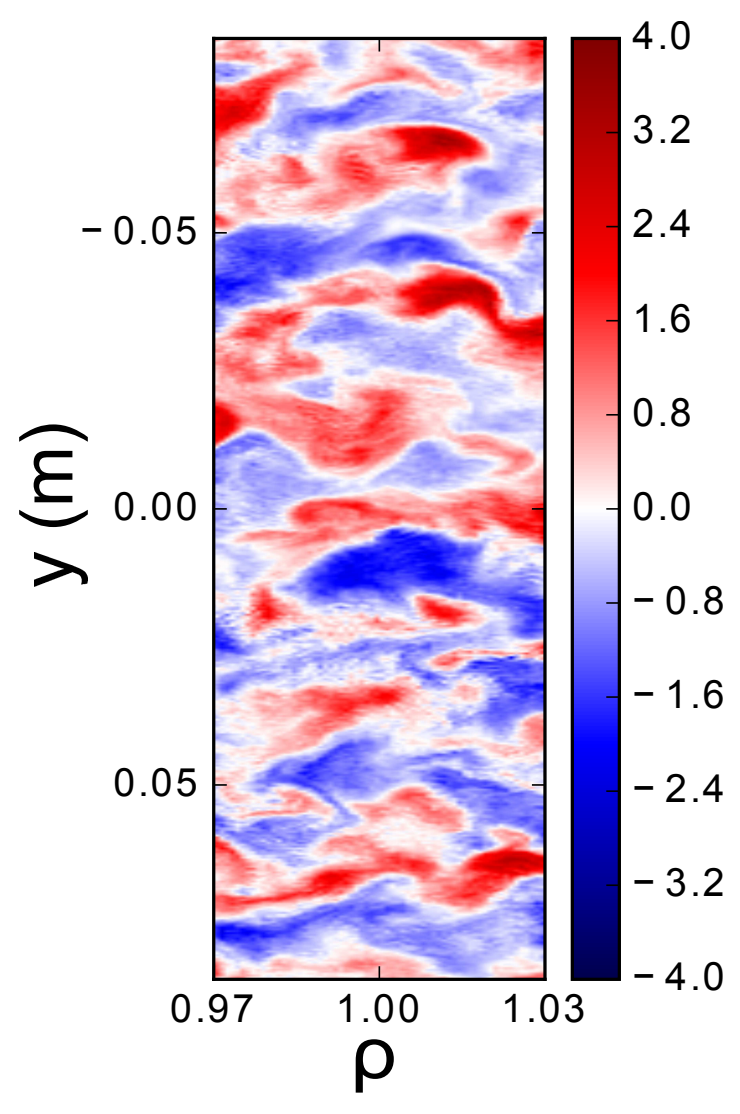

FIG. 2: Snapshot of the drift-plane at the outer midplane at high density $\left(n_{e}=3.4 \cdot 10^{19} \mathrm{~m}^{-3}\right)$.

are the collisionality $\hat{\nu}$ in units of $a / c_{s}$ and the plasma beta $\hat{\beta}=4 \pi n T_{e} / B^{2}$. The density variation corresponds to changes in $4.9 \leq \hat{\nu} \leq 78.4$ and $0.54 \cdot 10^{-5} \leq$ $\hat{\beta} \leq 8.58 \cdot 10^{-5}$. A transition in the normalized collisionality $\Lambda=\frac{L_{\|} / c_{s}}{1 / \nu_{e i}} \frac{\Omega_{i}}{\Omega_{e}}$ (with parallel connection length $L_{\|}$, ion sound speed $c_{s}$, electron-ion collision frequency $\nu_{e i} \sim \frac{n_{e}}{T_{e}^{3 / 2}}$, gyrofrequencies of ions and electrons $\Omega_{e, i}$ ) estimated from density and temperature one grid point ahead of the limiter boundary in parallel direction $\left(L_{\|} \approx\right.$ $3 \mathrm{~m}$ ) from below $\Lambda<1$ to above unity $\Lambda>1$ occurs around an edge density of $1 \cdot 10^{19} \mathrm{~m}^{-3}$ (Fig. 3). At this density also the shear layer (Fig. 4) gets strongly reduced. A reduction of the radial electric field at high Greenwald fraction as been also reported from HL-2A [36]. A transition of the dominant turbulence regime from drift-wave dominated to resistive ballooning dominated is expected to occur at $C \omega_{B}>1$ [37] which corresponds to $C>R / 2 L_{\perp} \approx 18.3$ with $L_{\perp}=4.5 \mathrm{~cm}$ and $R=1.65 \mathrm{~m}$. The local normalized collisionality is given by $C=0.51\left(m_{e} / m_{i}\right)\left(q R / L_{\perp}\right)^{2} \nu_{e}\left(L_{\perp} / c_{s}\right)$ and $\omega_{B}=2 L_{\perp} / R$ with electron and ion masses $m_{e}$ and $m_{i}$, respectively, safety factor $q$ and ion sound speed $c_{s}=\sqrt{T_{e} / m_{i}}$. This transition to resistive ballooning should occur at densities above $3 \cdot 10^{19} \mathrm{~m}^{-3}$ corresponding to an edge density above $60 \%$ of the Greenwald density.

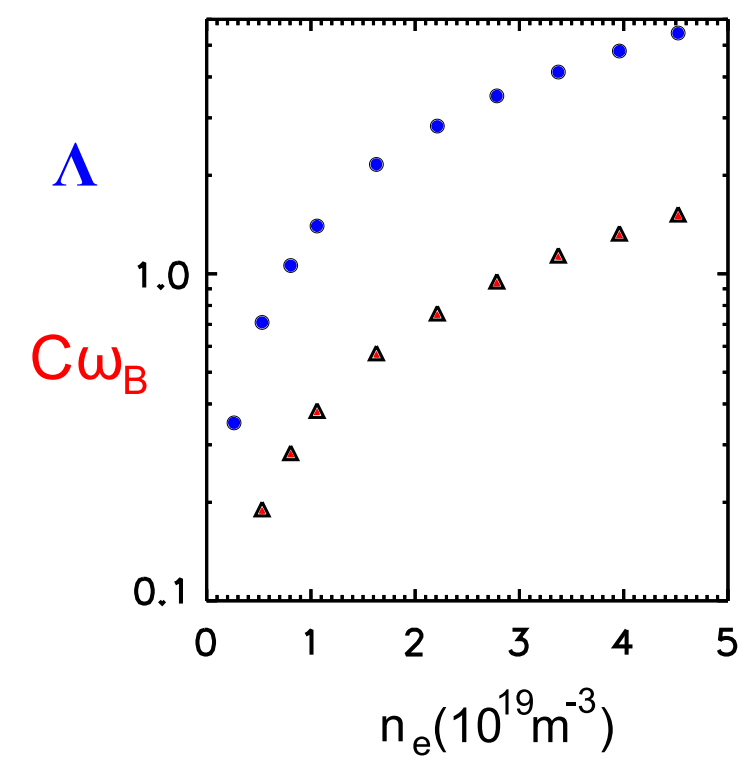

FIG. 3: Local collisionality $\Lambda$ in the limiter entrance as normalized by Myra et al. [35], and theoretically expected transition to resistive ballooning parameter $C \omega_{B}$.

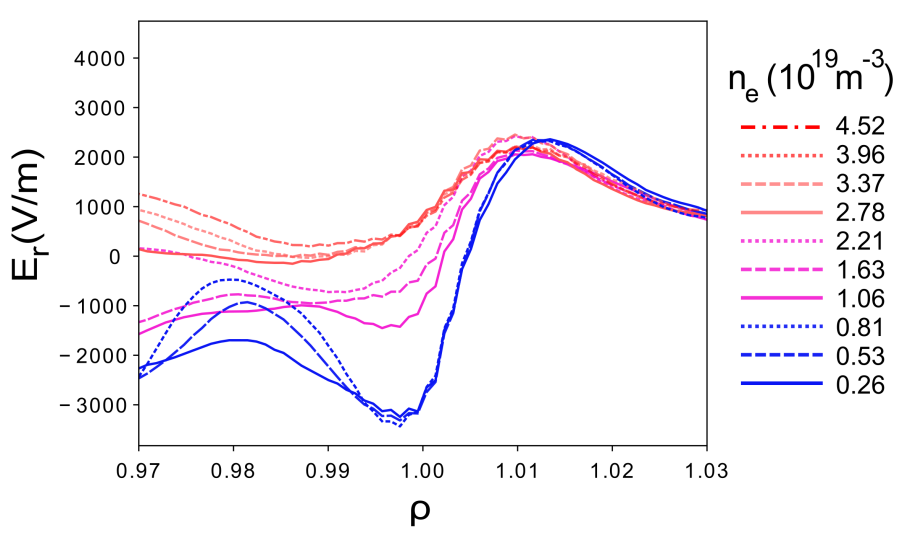

FIG. 4: The radial electric field changes strongly with increasing density. At low density the radial electric field is shown in a darker color than in for higher densities.

Above this transition $\left(C \omega_{B}>1\right)$ the well in the radial electric field is lost (Fig. 4). At these high densities the density limit occurs. These discharges are characterized by high radiation, which is not taken into account in the GEMR model. Although the presented simulation data set is not a complete realistic image of the transition of SOL turbulence at high densities, the development from seed fluctuation to plasma blobs around the separatrix should be qualitatively well represented by the simulations, providing an adequate testbed for the heuristic model presented in Ref. [12]. 


\section{BLOB TRACKING}

Basic characteristics of the blobs are estimated from the simulated data. For the purpose of determining the blob detection rate, single pixels in the detection region $(1.015 \leq \rho \leq 1.02)$ are utilized as detectors. To improve statistics, a whole row of pixels (512) is used. As soon as the critical threshold of $2.5 \sigma$ is exceeded, a detection event is registered. To avoid the detection of multiple events belonging to a single structure, an insensitive time of 100 time steps (about $37 \mu \mathrm{s}$ ) after each detection event is applied. Alternatively, a flexible insensitive time like in Ref. [38] can be used, where the detector becomes sensitive again, as soon as the density fluctuation drops below the mean density. Almost identical results are obtained from both methods and, hence, for simplicity, only those with the fixed insensitive time are shown. For low densities the blob detection rate decreases with increasing background density as in the experiments [38].

To characterize the blobs, an object recognition technique has been used. First, a threshold has been applied for each time frame, to mark regions with high density fluctuations as possible blobs. Connected areas marked as blobs are then detected by using a floodfill algorithm, which additionally gathers information used to calculate the centroid of the area. In order to obtain the blob size (FWHM) in y direction, the maximum value for the density fluctuation within the blob area along the centroid's radial position has been determined. Then, neighboring (in y direction) pixels are compared to get the full width of half maximum. The blob size increases with density. However, the increase is rather moderate compared to the experimental findings $[38,39]$. blob velocities have been obtained by tracking blobs over following frames. Between two successive frames, the detected blobs have been compared in position, size and velocity. Therefore, it has been possible to match them accordingly and to determine, where and when new blobs were formed, how they move and when they vanish. Once the blob trajectory has been obtained, the velocity is calculated from the difference in the centroid's positions and passed time between successive frames.

Qualitatively, the basic characteristics of the blobs as shown in Fig. 5 change similar to the experiment [38]. Fewer, larger and faster blobs are observed with increasing density. Compared to the experiment these changes are rather moderate. The blob size increases linearly by $30 \%$ with density up to $3 \cdot 10^{19} \mathrm{~m}^{-3}$ and saturates for larger densities, the velocity increases linearly by $150 \%$. The blob velocity is almost linear increasing with blob size, which points to the inertial regime of blob propagation taking into account the small variation in the blob size. In contrast to the experiment [39], no change in the propagation regime is observed. The comparison is not straightforward as the simulations cover only the near SOL and the experiments only the far SOL. The simulation domain covers only $2.5 \mathrm{~cm}$ in the SOL.

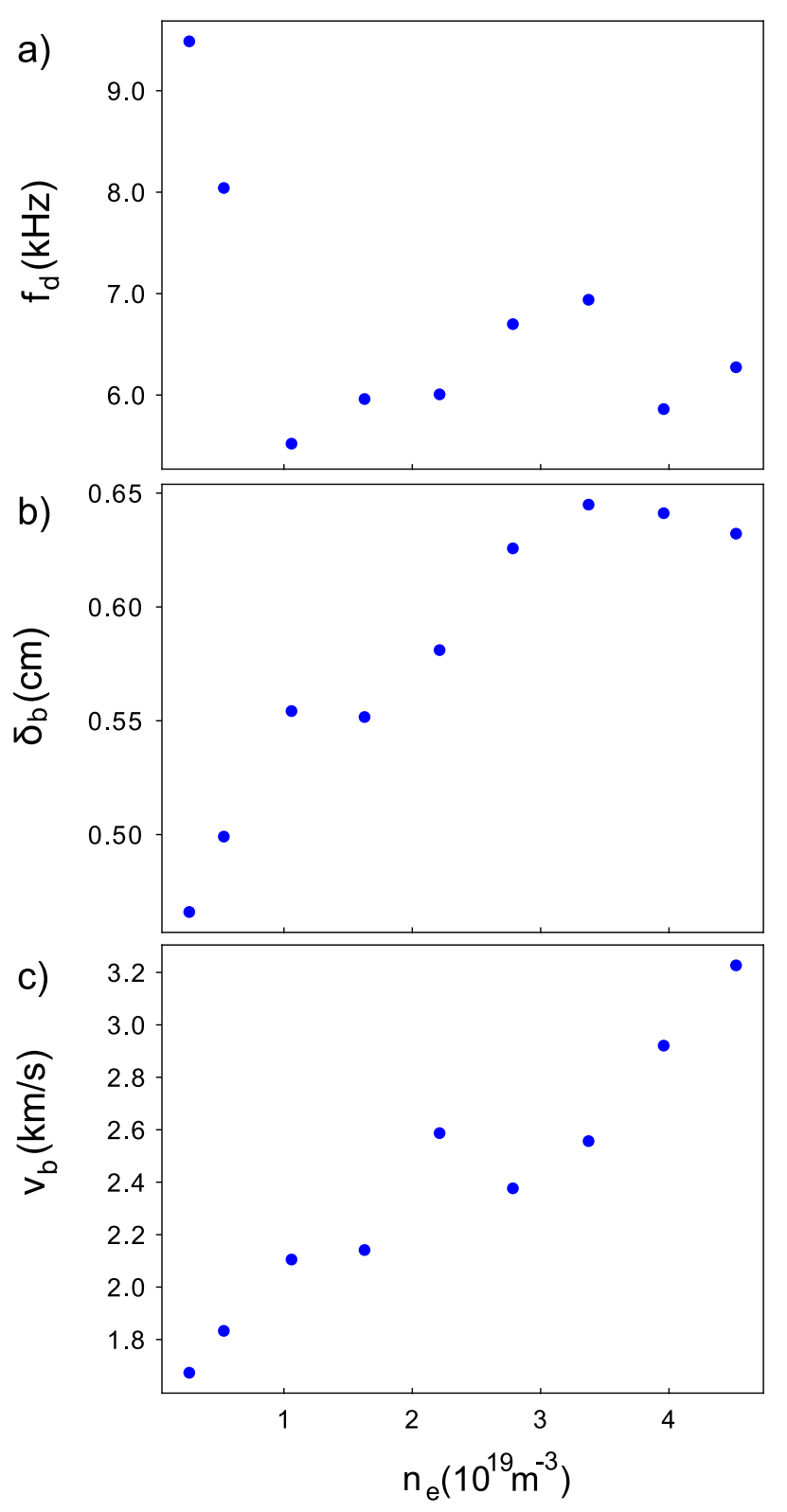

FIG. 5: Detection rate (a), size (b) and velocity (c) of blobs with varying background edge density.

\section{ROBUSTNESS OF BLOB SIZE}

In Ref. [12] it is assumed that a typical blob size exists, which is most stable against secondary instability. In the simulation different blobs sizes occur, the typical blob size $\delta_{b}$ is estimated as its mean value. How stable a particular blob size is can be rated by estimating a relative rate of change of the size by $\left(\Delta \delta_{b}\right) / \delta(b)=$ $\left\langle\left(\delta_{b}(t+1)-\delta_{b}(t)\right)^{2} / \delta_{b}(t)\right\rangle_{t}$ with time average $\langle\cdot\rangle_{t}$. Figure 6 a shows the rate of change of different blob sizes $\left(\Delta \delta_{b}\right)^{2} / \delta_{b}$ for the simulation at $n_{L C F S}=1.2 \cdot 10^{19} \mathrm{~m}^{-3}$ as an example shown by the blue crosses. The black line is a fit with a Gaussian kernel to guide the eye. The 

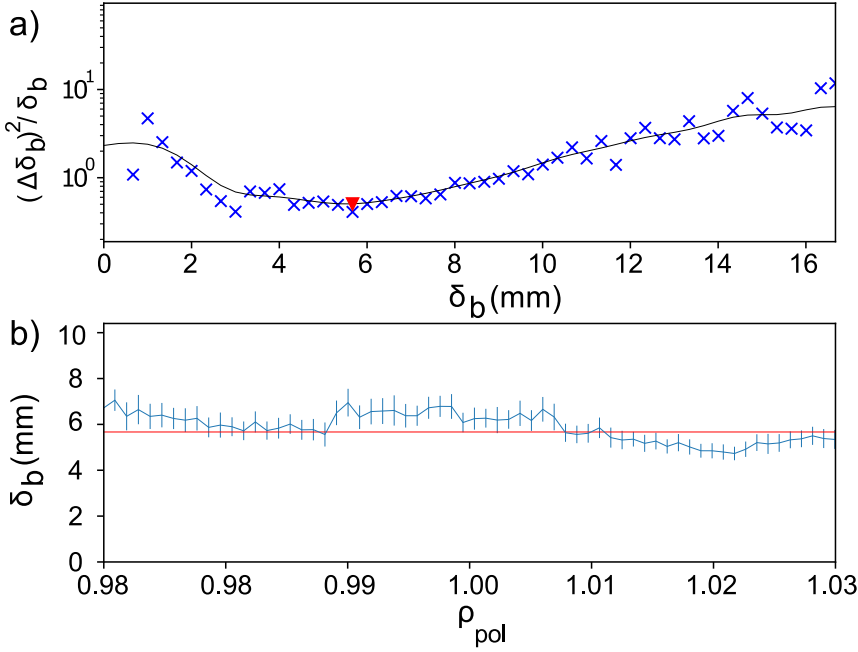

FIG. 6: Example (simulation with $n_{e}=1.06 \cdot 10^{19} \mathrm{~m}^{-3}$ for the rate of the change in the blob size in dependence of the blob size shown by the blue crosses (upper figure). The black line is a fit with a Gaussian Kernel. The most stable blob size is indicated by the red triangle. The lower figure shows a comparison of the most stable blob size (red) with the mean blob size (blue) over a range of radial positions.

red triangle is located at the fitted curve's minimum and therefore marking the most stable blob size. A comparison between the mean size (blue) for a range of radial positions is shown in Fig. $6 \mathrm{~b}$ and compared to the most stable blob size (red) determined from Fig. 6a. The error bars in Fig. $6 \mathrm{~b}$ are calculated by the standard deviation $\sigma_{\delta_{b}}$, which is additional weighted by the number $N$ of measurements of blobs. It can be concluded, that the mean blob size stays nearly constant over the radius and both values are in good agreement with each other. Only moderate changes in the typical blob size are observed over the simulation region. This behavior is found for all presented simulations. Therefore, this assumption seems to be valid to the first order. In experiments in ASDEX Upgrade the blob size appears also rather constant over the radius except for very large blobs $\left(\delta_{b}>5 \mathrm{~cm}\right)$ at high densities [38].

\section{BLOB GENERATION}

\section{A. Blob tracking}

The detection line has been set to $\rho_{\text {pol }}=1.015$ or $\rho=1.020$, respectively. To estimate the blob birth region all trajectories crossing the detection line are traced back to their origin. This is called the direct method in the following. In the present simulations blobs are not generated at one particular position and most of the blobs originate from a region outside the confined region. Blobs preferentially generated outside the confined a)

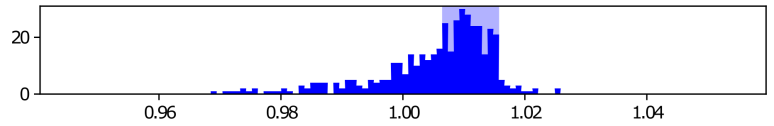

b)

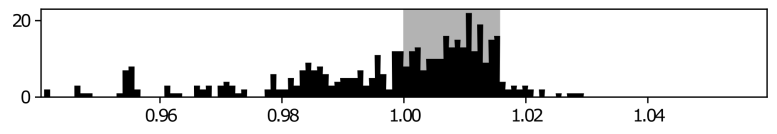

c)

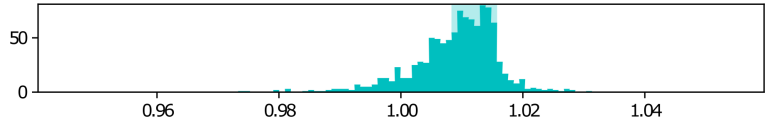

d)

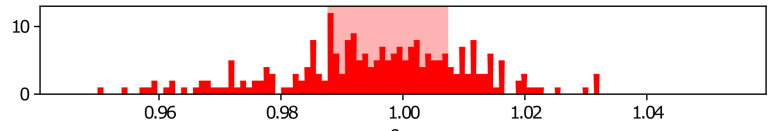

e)

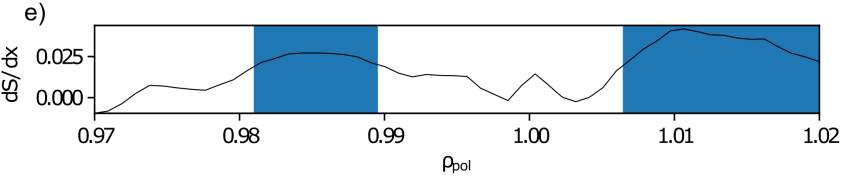

FIG. 7: Histograms of blob generation position for one simulation as an example $\left(n_{L C F S}=1.2 \cdot 10^{19} \mathrm{~m}^{-3}\right)$ by using different methods: direct blob tracking (a), family tree (b), blob tracking of ion temperature fluctuations (c), fitting the blob ion temperature to the background value (d), rise in skewness (e).

region have been reported from NSTX [40]. A physical explanation for blobs preferentially generated outside the confined region can be found in Refs. [34, 41]. For the following procedure the generation area is defined by the smallest possible region in which $50 \%$ of all trajectories originate from. To detect possible seed fluctuations, which appear at a lower amplitude, the critical threshold for blob detection has been varied between $0.5 \sigma$ and $2.5 \sigma$ in $0.25 \sigma$ steps and the generation region estimated in this way does not change and is therefore independent of the critical threshold. This method estimates the blob generation region outside the LCFS for all simulations (Fig. 8). The reason might be the strong interaction of the blobs in the shear layer region, which lead to slitting and merging of blobs [34]. By investigating the raw data it is obvious that the blobs undergo multiple interactions with each other and with the background shear flow before they actually propagate just radially.

After the blob generation position has been estimated, the velocity of the blobs in the binormal direction corresponding to $v_{E x B}+v_{p h}$ and the blob size $\delta_{b}$ are estimated at this position. This position is always a region over which the quantities are integrated. The blob generation rate $f_{g}$ is calculated following Eq. (1) estimated at the generation region. The blob detection rate $f_{d}$ is estimated in a predefined detection line, which has been set to $\rho_{\text {pol }}=1.015$ or $\rho=1.020$, respectively. For the comparison of generation and detection rate (Eq. (2)), $\gamma_{d}$ has been evaluated following Eq. (3) using the den- 


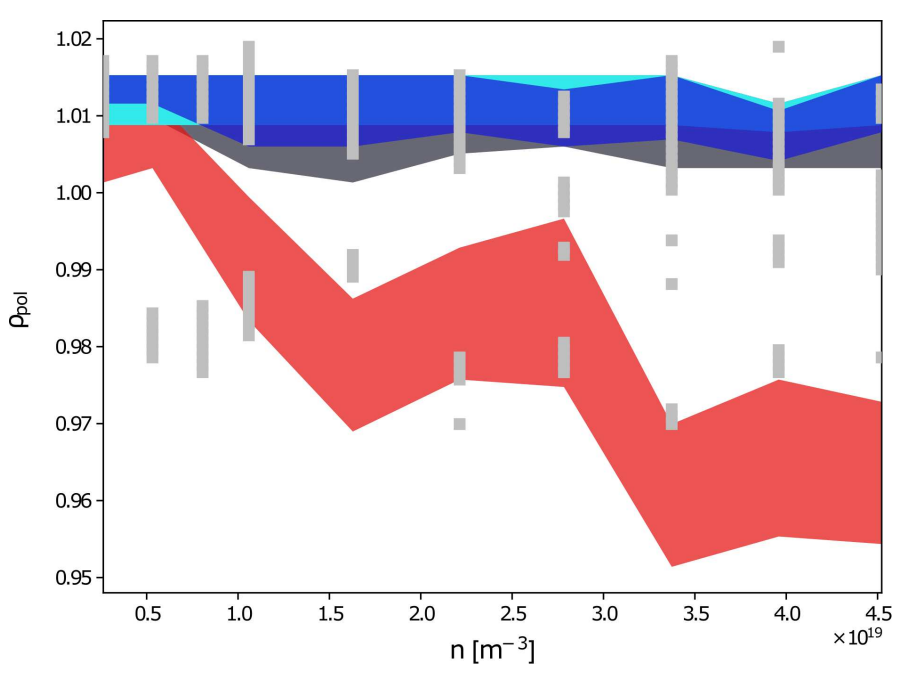

FIG. 8: Blob generation region for different densities at the LCFS estimated with different methods: direct blob tracking (blue), family tree (black), blob tracking of ion temperature fluctuations (cyan), fitting the blob ion temperature to the background value (red), rise in skewness (grey).

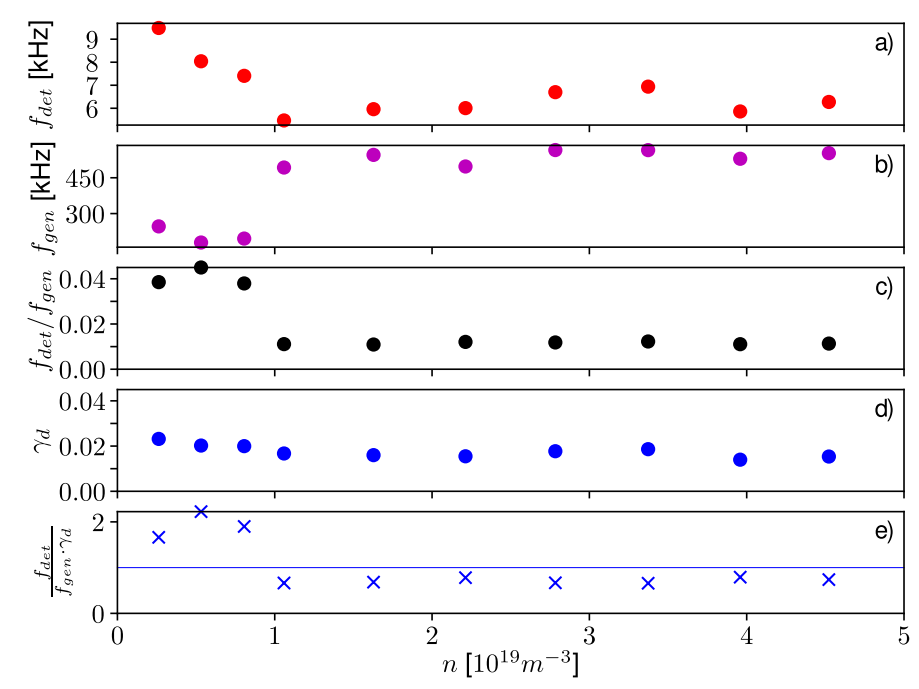

FIG. 9: Blob detection rate $f_{d}(\mathrm{a})$, generation rate $f_{g}(\mathrm{~b})$, its ratio $f_{d} / f_{g}(\mathrm{c}), \gamma_{d}(\mathrm{~d})$ and $f_{d} /\left(\gamma_{d} f_{g}\right)$ for blob birth region estimated by tracing back blob trajectories from the detection region $\rho=1.02$.

sity fluctuations at the generation position. The result from the detector position at $\rho=1.020$ is shown in Fig. 9. For low densities $\left(n_{e}<10^{19} \mathrm{~m}^{-3}\right)$ the blob occurrence rate $f_{d}$ is underestimated by $\gamma_{d} f_{g}$, for high densities $\left(n_{e}>10^{19} \mathrm{~m}^{-3}\right)$ it is overestimated. Such anticorrelation may indicate a breakdown of the model prediction. Some overprediction by the model prediction occurs due to the lack of taking blob dissipation into account. Some of the blobs will be dissipated on the way from their generation position to the detection position.
The underprediction at low densities is more disconcerting. The transition is accompanied by a strong reduction of the $E_{r}$ well and the corresponding strong reduction of the outer shear layer (Fig. 4). At low densities with a strong shear, a generation process is enabled, who's mechanism is based on merging blobs inside and outside the shear layer. Thereby, a density transfer into the SOL is archived [34]. The blob generation rate hereby depends on the merging rate and hence, on the relative velocity of adjacent layers, as well as the rate of blobs moving from the confinement region into the shear layer. A blob moving into the shear layer can undergo multiple merging and splitting events. Taking into account only the blobs generated outside the confinement leads to an underestimation.

\section{B. Blob tracking including a family tree}

With this method effects like slitting and merging are taken into account. As in the direct methods blobs are traced back to their origin. The origin of a blob is compared to the last position of other blob trajectories. If a newly generated blob overlaps with the area of another blob, which had dissipated only a few time steps before, or shows a displacement of 5 or less pixels from it's position, both blobs are considered to be of one family. By taking these effects into account the generation region is moving a bit inside (Figs. 7b and 8) but shows roughly the same results as the direct method.

\section{Ion temperature method}

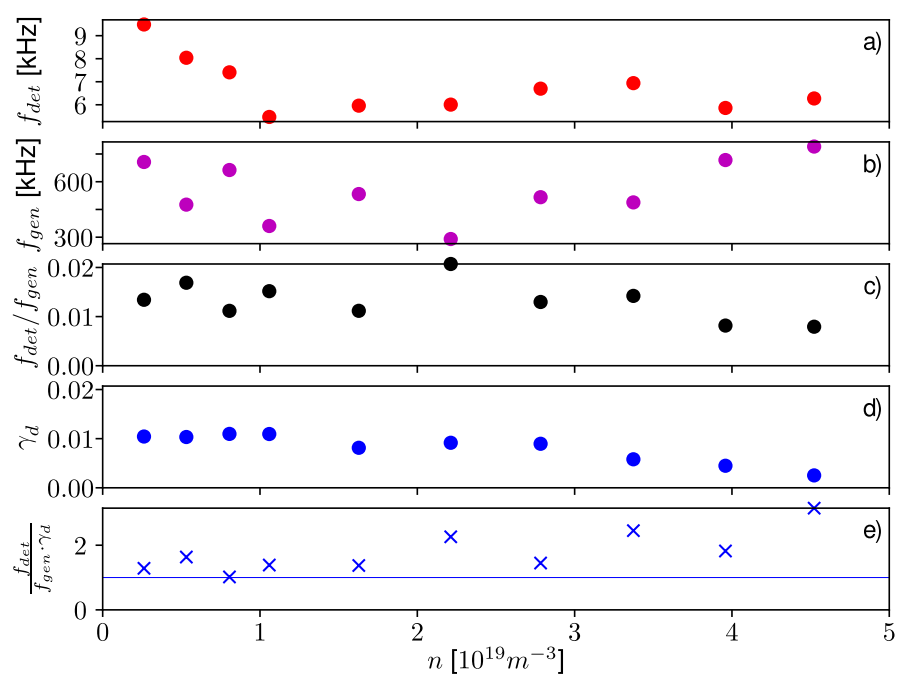

FIG. 10: Blob detection rate $f_{d}$ (a), generation rate $f_{g}(\mathrm{~b})$, its ratio $f_{d} / f_{g}(\mathrm{c}), \gamma_{d}(\mathrm{~d})$ and $f_{d} /\left(\gamma_{d} f_{g}\right)$ for blob birth region estimated by matching background ion temperature with blob ion temperature at the detection region $\rho=1.02$. 
Another method to estimate the generation region of the seed fluctuation has been proposed in Refs. [42, 43]. It is assumed that the ion temperature of the turbulent structures is not changing much during their propagation even if they undergo multiple interactions. It is assumed that the location of the seed fluctuations is at the position, where the mean ion temperature profile matches the blob ion temperature. It is observed that the region, where the ion background temperature matches the ion temperature of the blobs moves inside with increasing density (Fig. 8). At low density both positions are close to each other. At higher background densities the region determined by the ion temperature is within the confined region. The detection rate exceeds the model prediction (Fig. 10e). The discrepancy increases with density as the blob birth regions moves inside. At very high densities the region, where the background ion temperature matches the blob ion temperature, moves very close to the inner boundary of the simulations. This will possibly lead to some discrepancies as the boundary effects may influence the results (for example the drop of $\gamma_{d}$ below 0.01 (Fig. 10d)). The discrepancy between detection rate and model prediction can be either ascribed to the short comings of the model [12] or to a non representative blob birth region.

Also the direct method has been applied to the ion temperature fluctuation field. As shown in Figs. 7c and 8 the results show similar results as the direct method for the density fluctuations. Therefore, there is some doubt if the structures are more persistent in the ion temperature compared to the density.

\section{Rise in skewness}

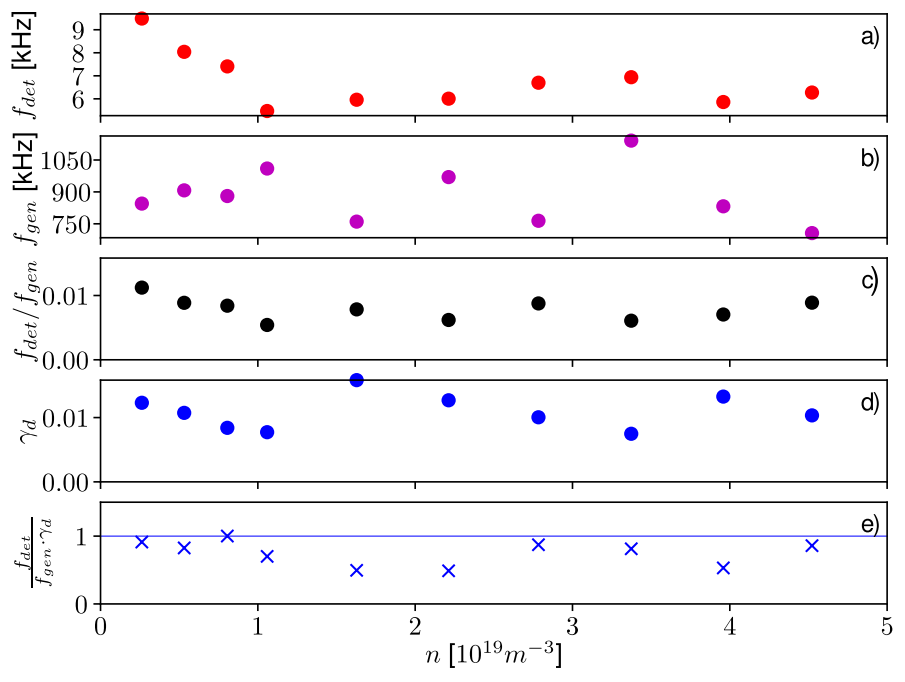

FIG. 11: Blob detection rate $f_{d}$ at detection region $\rho=1.02$ (a), generation rate $f_{g}(\mathrm{~b})$, its ratio $f_{d} / f_{g}(\mathrm{c}), \gamma_{d}(\mathrm{~d})$ and $f_{d} /\left(\gamma_{d} f_{g}\right)$ for blob birth region estimated by the strongest rise in skewness.
Usually the skewness is taken to determine the blob birth position. As blobs are indicated by large positive events, which is associated to a large skewness, the position where the skewness changes from positive (blob dominated) to negative (hole dominated) might be seen as the birth position. However, as shown in Ref. [41] this does not indicate the position, where most of the blobs are generated. Instead it has been proposed to use the position of the strongest increase in the skewness. The maximum increase in skewness has been estimated as shown in Fig. 7e. These positions are inside and outside of the outer shear layers, also inside and outside of the confined region as shown in Fig. 8. Both of the regions where the skewness gradient maximizes are taken into account. By splitting up the generation region the overall agreement between detection rate and model prediction (Fig. 11e) is improved compared to the other approaches discussed above.

\section{SUMMARY AND CONCLUSION}

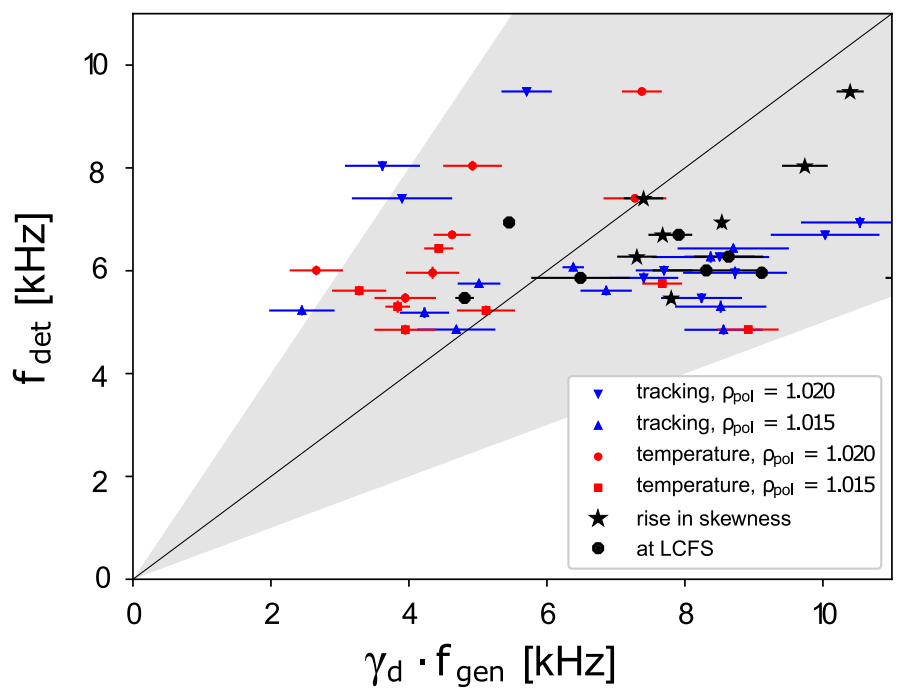

FIG. 12: Blob detection rate $f_{d}$ in dependence of its prediction $\gamma_{d} f_{g}$. Error bars in $f_{d}$ are smaller than the points.

The predictive capability of a recent model for the prediction of the blob detection rate [12] have been studied by means of gyrofluid simulations. Some aspects of the blob generation process may be worthwhile to point out first. There exists a typical blob size, which stays rather constant across the region of propagation across the separatrix and the near SOL. The most problematic assumption seems to be the primal region of generation. It is found that blobs are not generated at one particular position. Different methods have been used to define the primal blob generation position. Tracing back the trajectories of the blobs to their origin, blobs are generated mostly just outside the last closed flux surface. Matching 
the ion temperature of the blobs to the background ion temperature implies an inward shift from just outside the last closed flux surface at low densities into the confined region with increasing density.

The best agreement (qualitatively and quantitatively as well) is found for the region determined by the strongest rise in skewness. However, it should be noted that using the LCFS position directly also shows a good agreement and can be used as a first estimate. Despite varying generation positions corresponding to different driving instabilities and background flow velocities, a comparison of the blob occurrence rate with the prediction shows an agreement within a factor of two (summarized in in Fig. 12). This agreement is remarkably good keeping in mind the simplicity of the approach in Ref. [12]. Therefore, the model seems to be able to predict or, at least, estimate the blob occurrence rate.
For experimental tests of the model it is recommended to use the rise in skewness to determine the blob generation region or regions, at which the amplitude distribution function can be obtained directly and the total binormal velocity ( $E \times B$ and phase velocity) can be measured by a time delay estimation method [44].

\section{Acknowledgements}

This work has been carried out within the framework of the EUROfusion Consortium and has received funding from the Euratom research and training programme 2014-2018 under grant agreement No 633053. The views and opinions expressed herein do not necessarily reflect those of the European Commission.
[1] J. Boedo, D. Rudakov, R. Moyer, S. Krasheninnikov, D. Whyte, G. McKee, G. Tynan, M. Schaffer, P. Stangeby, P. West, et al., Phys. Plasmas 8, 4826 (2001).

[2] Y. H. Xu, S. Jachmich, and R. R. Weynats, Plasma Phys. \& Controlled Fusion 47, 1841 (2005).

[3] J. Cheng, L. Yan, W. Hong, T. Lan, J. Qian, A. Liu, H. Zhao, Y. Liu, Q. Yang, J. Dong, et al., Plasma Phys. \& Controlled Fusion 52, 055003 (2010).

[4] S. Krasheninnikov, Phys. Lett. A 283, 368 (2001).

[5] O. E. Garcia, J. Horacek, R. A. Pitts, A. H. Nielsen, W. Fundamenski, J. P. Graves, V. Naulin, and J. J. Rasmussen, Plasma Physics and Controlled Fusion 48, L1 (2006).

[6] P. Manz, D. Carralero, G. Birkenmeier, H. W. Müller, S. H. Müller, G. Fuchert, B. D. Scott, and U. Stroth, Phys. Plasmas 20, 102307 (2013).

[7] R. Kube and O. E. Garcia, Phys. Plasmas 18, 102314 (2011).

[8] G. Birkenmeier, F. M. Laggner, M. Willensdorfer, T. Kobayashi, P. Manz, E. Wolfrum, D. Carralero, R. Fischer, B. Sieglin, G. Fuchert, et al., Plasma Phys. \& Controlled Fusion 56, 075019 (2014).

[9] G. Fuchert, G. Birkenmeier, D. Carralero, T. Lunt, P. Manz, H. W. Müller, B. Nold, M. Ramisch, V. Rohde, U. Stroth, et al., Plasma Phys. \& Controlled Fusion 56, 125001 (2014)

[10] N. R. Walkden, B. D. Dudson, L. Easy, G. Fishpool, and J. T. Omotani, Nucl. Fusion 55, 113022 (2015).

[11] J. Olsen, J. Madsen, N. A. H., J. J. Rasmussen, and V. Naulin, Plasma Phys. \& Controlled Fusion 58, 044011 (2016).

[12] G. Fuchert, D. Carralero, P. Manz, U. Stroth, E. Wolfrum, and the ASDEX Upgrade Team, Plasma Phys. \& Controlled Fusion 58, 054006 (2016).

[13] N. Fedorczak, J. P. Gunn, N. Nace, A. Gallo, C. Baudoin, H. Bufferand, G. Ciraolo, T. Eich, P. Ghendrih, and P. Tamain, Nucl. Mat. and Energy 12, 838 (2017).

[14] G. Birkenmeier, P. Manz, D. Carralero, F. M. Laggner, G. Fuchert, K. Krieger, H. Maier, F. Reimold, K. Schmid, R. Dux, et al., Nucl. Fusion 55, 033018 (2015).
[15] R. Wenninger, R. Albanese, R. Ambrosino, F. Arbeiter, J. Aubert, C. Bachmann, L. Barbato, T. Barrett, M. Beckers, W. Biel, et al., Nucl. Fusion 57, 046002 (2017).

[16] J. Madsen, O. E. Garcia, J. S. Larsen, V. Naulin, A. H. Nielsen, and J. J. Rasmussen, Phys. Plasmas 18, 112504 (2011).

[17] D. A. D'Ippolito and J. R. Myra, Phys. Plasmas 10, 4029 (2003).

[18] A. Y. Aydemir, Phys. Plasmas 12, 062503 (2005).

[19] B. Labit, I. Furno, A. Fasoli, A. Diallo, S. H. Müller, G. Plyushchev, M. Podestà, and F. M. Poli, Phys. Rev. Lett. 98, 255002 (2007).

[20] F. Sattin, M. Agostini, P. Scarin, N. Vianello, R. Cavazzana, L. Marrelli, G. Serianni, S. J. Zweben, R. J. Maqueda, Y. Yagi, et al., Plasma Physics and Controlled Fusion 51, 055013 (2009).

[21] H. Zushi, N. Nishino, K. Hanada, H. Honma, H. Liu, Y. Higashizono, M. Sakamoto, S. Tashima, and T. Ryoukai, Journal of Nuclear Materials 415, S624 (2011).

[22] B. Nold, G. D. Conway, T. Happel, H. Müller, M. Ramisch, V. Rohde, U. Stroth, and ASDEX Upgrade Team, Plasma Phys. \& Controlled Fusion 52, 065005 (2010).

[23] G. S. Xu, V. Naulin, W. Fundamenski, C. Hidalgo, J. A. Alonso, C. Silva, B. Gonalves, A. H. Nielsen, J. J. Rasmussen, S. Krasheninnikov, et al., Nuclear Fusion 49, $092002(2009)$.

[24] B. LaBombard, J. W. Hughes, D. Mossessian, M. Greenwald, B. Pipschultz, J. L. Terry, and the Alcator C-Mod Team, Nucl. Fusion 45, 1658 (2005).

[25] O. E. Garcia, S. M. Fritzner, R. Kube, I. Cziegler, B. LaBombard, and J. L. Terry, Phys. Plasmas 20, 055901 (2013).

[26] B. D. Scott, Contrib. Plasma Phys. 46, 714 (2006).

[27] A. Kendl, B. D. Scott, and T. T. Ribeiro, Phys. Plasmas 17, $072302(2010)$.

[28] S. J. Zweben, B. D. Scott, J. L. Terry, B. LaBombard, J. W. Hughes, and D. P. Stotler, Phys. Plasmas 16, 082505 (2009).

[29] M. Kočan, F. P. Gennrich, A. Kendl, H. W. Müller, and 
ASDEX Upgrade Team, Plasma Phys. \& Controlled Fusion 54, 085009 (2012).

[30] B. Nold, T. T. Ribeiro, M. Ramisch, Z. Huang, H. W. Müller, B. D. Scott, U. Stroth, and ASDEX Upgrade Team, New. J. Phys. 14, 063022 (2012).

[31] P. Manz, G. Birkenmeier, D. Carralero, G. Fuchert, H. W. Müller, S. H. Müller, B. D. Scott, U. Stroth, T. T. Ribeiro, E. Wolfrum, et al., Plasma Phys. \& Controlled Fusion 57, 014012 (2015).

[32] T. T. Ribeiro and B. Scott, Plasma Phys. \& Controlled Fusion 47, 1657 (2005).

[33] T. T. Ribeiro and B. Scott, Plasma Phys. \& Controlled Fusion 50, 055007 (2008).

[34] B. Nold, P. Manz, T. T. Ribeiro, G. Fuchert, G. Birkenmeier, H. W. Müller, M. Ramisch, B. D. Scott, and U. Stroth, Phys. Plasmas 21, 102304 (2014).

[35] J. R. Myra, D. A. Russell, and D. A. D'Ippolito, Physics of Plasmas 13, 112502 (2006).

[36] R. Hong, G. R. Tynan, P. H. Diamond, L. Nie, D. Guo, T. Long, R. Ke, Y. Wu, B. Yuan, M. Xu, et al., Nucl. Fusion p. 016041 (2018).

[37] B. D. Scott, Phys. Plasmas 12, 062314 (2005).

[38] D. Carralero, G. Birkenmeier, H. W. Müller, P. Manz, P. deMarne, S. Müller, F. Reimhold, U. Stroth, M. Wischmeier, E. Wolfrum, et al., Nucl. Fusion 54, 123005
(2014).

[39] D. Carralero, P. Manz, L. Aho-Mantila, G. Birkenmeier, M. Brix, H. W. Müller, U. Stroth, N. Vianello, E. Wolfrum, and the ASDEX Upgrade Team, Phys. Rev. Lett. 115, $215002(2015)$

[40] J. R. Myra, D. A. D'Ippolito, D. P. Stotler, S. J. Zweben, B. P. LeBlanc, J. E. Menard, R. J. Maqueda, and J. Boedo, Physics of Plasmas 13, 092509 (2006).

[41] P. Manz, T. T. Ribeiro, B. D. Scott, G. Birkenmeier, D. Carralero, G. Fuchert, H. W. Müller, S. H. Müller, U. Stroth, and E. Wolfrum, Phys. Plasmas 22, 022308 (2015).

[42] A. H. Nielsen, J. Rasmussen, J. Madsen, G. S. Xu, V. Naulin, J. Olsen, M. Loiten, S. Hansen, N. Yan, L. Tophoj, et al., Plasma Phys. \& Controlled Fusion 59, 025012 (2017).

[43] D. Carralero, M. Siccino, M. Komm, S. A. Artene, F. D. Isa, J. Adamek, L. Aho-Mantila, G. Birkenmeier, M. Brix, G. Fuchert, et al., Nucl. Fusion 57, 056044 (2017).

[44] N. Fedorczak, P. Manz, S. C. Thakur, M. Xu, G. R. Tynan, G. S. Xu, and S. C. Liu, Phys. Plasmas 19, 122302 (2012). 